\title{
Nonbinary LDPC Codes for Optical Communication Systems
}

\author{
Ivan B. Djordjevic, Member, IEEE, and Bane Vasic, Senior Member, IEEE
}

\begin{abstract}
We propose a class of moderate-length nonbinary low-density parity-check (LDPC) codes for long-haul high-speed optical transmission systems. In particular, we present an LDPC code with $12.59 \%$ redundancy that achieves a 9.9-dB coding gain at a bit-error rate of $10^{-10}$. A similar coding gain is obtained for a long nonbinary LDPC code with a redundancy of only $6.7 \%$.
\end{abstract}

Index Terms-Forward-error correction (FEC), GF $(q)$, lowdensity parity-check (LDPC) codes, optical communications.

\section{INTRODUCTION}

$\mathbf{T}$ HE standard forward-error-correction (FEC) scheme of fiber-optics communication systems is based on Reed-Solomon (RS) codes: $(255,238)$ RS code in G.709 standard, and $(255,239) \mathrm{RS}$ code in G.975 standard [1], [2]. Optical transmission systems at high bit rates ( $40 \mathrm{~Gb} / \mathrm{s}$ and above) face numerous challenges including: 1) increase in intrachannel nonlinearities, 2) stringent polarization-mode dispersion requirements, 3) unavailability of commercial high-speed electronics for high-speed transmitters, and 4) increased demands in chromatic dispersion accuracy. In order to overcome these challenges, novel FEC schemes providing large coding gains at high code rates are highly desirable.

Iteratively decodable codes, turbo [3], [4], and low-density parity-check (LDPC) [5]-[15] codes have generated significant research attention recently. The coding gains demonstrated in an additive white Gaussian noise (AWGN) channel [3] and our results for optical communication channel [5], [6], [9]-[11] show that such codes are a viable choice for $40-\mathrm{Gb} / \mathrm{s}$ transmission. Unfortunately, the capacity approaching coding gains can only be achieved by employing extremely long binary LDPC codes, which would introduce an unacceptable processing delay in the decoder. On the other hand, from theoretical results in [7] and [8], it follows that codes over higher order Galois fields may be able to provide large coding gains even for moderate lengths.

This letter investigates the performance of regular LDPC codes, constructed over binary extension fields of $\mathrm{GF}(2)$, on optical communication channel at $40 \mathrm{~Gb} / \mathrm{s}$. The bit-error-rate (BER) performance of these codes obtained via simulations show that: 1) an $\operatorname{LDPC}(1225,1088)$ code of moderate length with $12.59 \%$ redundancy over $\mathrm{GF}(8)$ achieves a coding gain of $9.9 \mathrm{~dB}$ at BER of $10^{-10}$, and 2) an $\operatorname{LDPC}(29136,27315)$ with $6.7 \%$ redundancy achieves a coding gain of $9.7 \mathrm{~dB}$ at BER

Manuscript received April 19, 2005; revised July 3, 2005. This work is supported by the NSF under Grant ITR 0325979 and Grant CCR 0208597.

The authors are with the Department of Electrical and Computer Engineering, University of Arizona, Tucson, AZ 85721 USA (e-mail: ivan@ece.arizona.edu; vasic@ece.arizona.edu).

Digital Object Identifier 10.1109/LPT.2005.856337 of $10^{-10}$, which is comparable to the best turbo product code proposal [4] with $24.6 \%$ redundancy.

\section{NONBINARY LDPC CODES}

Nonbinary LDPC codes are linear block codes described by a sparse parity-check matrix $\boldsymbol{H}$ over a Galois field $\operatorname{GF}(q)$. A straightforward way of generating an $\boldsymbol{H}$ matrix is to randomly generate a sparse binary matrix [7] and to replace the nonzero entries with randomly chosen nonzero elements of $\mathrm{GF}(q)$. Another approach, proposed in [7], is to choose the entries of $\boldsymbol{H}$ using a carefully selected random distribution of elements from $\mathrm{GF}(q)$. However, the Tanner graph of nonstructured nonbinary LDPC codes is too complicated for practical high-speed applications, and therefore, we restrict our attention to structured LDPC codes.

In this letter, we consider two classes of regular LDPC codes over binary extension fields $\operatorname{GF}(q)$, where $q=2^{s}$ : 1) the nonzero entries in binary structured $\boldsymbol{H}$ matrix are replaced by randomly selected nonzero elements from $\operatorname{GF}\left(2^{s}\right)$, and 2) the nonbinary parity-check matrix is designed in a block-circulant fashion. Two codes from the first class, both based on combinatorial objects known as mutually orthogonal Latin rectangles [6], are considered: girth-6 codes designed using the MacNeish-Mann theorem [5], and girth-8 codes designed using the algorithm we proposed in [6]. As an illustrative example, the following parity-check matrix is obtained by replacing the ones in the binary matrix, designed using the MacNeish-Mann theorem, by randomly selected nonzero elements from $\operatorname{GF}(4)=\left\{0,1, \alpha, \alpha^{2}\right\}$

$$
H=\left[\begin{array}{ccccccccc}
1 & 0 & 0 & 0 & 0 & \alpha^{2} & 0 & 1 & 0 \\
0 & 1 & 0 & 1 & 0 & 0 & 0 & 0 & \alpha^{2} \\
0 & 0 & 1 & 0 & 1 & 0 & 1 & 0 & 0 \\
1 & 0 & 0 & 0 & \alpha^{2} & 0 & 0 & 0 & \alpha^{2} \\
0 & 0 & \alpha & 1 & 0 & 0 & 0 & 1 & 0 \\
0 & \alpha & 0 & 0 & 0 & \alpha & 1 & 0 & 0
\end{array}\right]
$$

The parity-check matrix of a regular nonbinary block-circulant LDPC code can be written as

$H=\left[\begin{array}{cccc}\alpha^{0} P^{i_{1}} & \alpha^{1} P^{i_{2}} & \ldots & \alpha^{l-1} P^{i_{l}} \\ \alpha^{l-1} P^{i_{l}} & \alpha^{0} P^{i_{1}} & \ldots & \alpha^{l-2} P^{i_{l-1}} \\ \ldots & \ldots & \ldots & \ldots \\ \alpha^{l-r+1} P^{i_{l-r+2}} & \alpha^{l-r+2} P^{i_{l-r+3}} & \ldots & \alpha^{l-r} P^{i_{l-r+1}}\end{array}\right]$

where $P$ is an $N \times N$ permutation matrix defined by [9]. The $\alpha^{i}, i=0,1, \ldots, l-1$, denotes a nonzero element of $\operatorname{GF}(q)$. For $l>q-2$ the multipliers from (1) are repeated $\lfloor l /(q-1)\rfloor$ times, since for the multiplicative group of $\operatorname{GF}(q) \alpha^{q-1}=1$. The exponents in (1) are selected as elements from the following set: 


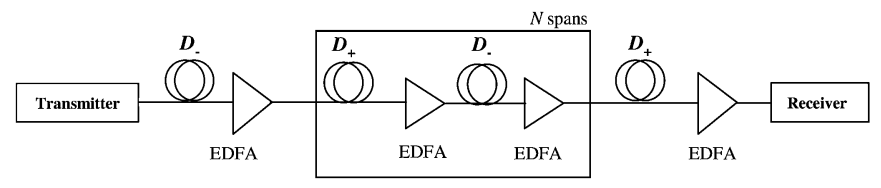

Fig. 1. Dispersion map under study.

$L=\left\{i: 0 \leq i \leq p^{2}-1, \theta^{i}+\theta \in \mathrm{GF}(p)\right\}$, where $\theta$ is the primitive element of the finite field $\operatorname{GF}\left(p^{2}\right)$. Notice that the finite field of order $p$ ( $p$-prime) is used to generate the set $L$ in the set. The parity-check matrix of a nonbinary block-circulant LDPC code (1) is in fact the generalization of our recent proposal in [9]. The primitive element $\theta$ is chosen in such a way that the set $L$ is a cyclic-invariant difference set (see [9]).

A block of $k s$ information bits is converted to a sequence of $k$ symbols, which forms an information word $\boldsymbol{b}$ with elements in $\operatorname{GF}\left(2^{s}\right)$. The information word $\boldsymbol{b}$ is encoded to the codeword $\boldsymbol{c}=\boldsymbol{b} G$, where $\boldsymbol{G}$ denotes the generator matrix. The binary form of the codeword $\boldsymbol{c}$ is transmitted through the channel. The decoding problem is to find the most likely vector $\hat{\boldsymbol{c}}$ satisfying the syndrome equation, with initial likelihood being determined by the channel model. The received vector $\boldsymbol{x}$ is converted to probabilities [7] or to log-likelihood ratios (LLRs) [8], depending on the implementation of the sum-product algorithm (SPA).

\section{System DescriPtion AND PeRformanCe ANALysis}

The system of interest in this letter is a wavelength-divisionmultiplexing (WDM) system. The continuous-wave laser signals at different wavelengths are modulated using independently encoded electrical streams and a Mach-Zehnder (MZ) modulator, WDM multiplexed and transmitted over the same fiber. The carrier-suppressed return-to-zero modulator employed is composed of a laser diode, two MZ intensity modulators, a pseudorandom binary sequence generator, and an encoder. The optical signal at the receiver side is split into separate channels by using an optical demultiplexer. Erbium-doped fiber amplifiers and dispersion-compensating fibers (DCF or $D_{-}$fiber) are deployed periodically to compensate the loss and accumulated dispersion of the standard single-mode fiber (SMF or $D_{+}$fiber). The propagation of a signal through the transmission media is modeled by a nonlinear Schrödinger equation [10] solved using the split-step Fourier method. The effects of both interchannel (cross-phase modulation (XPM), four-wave mixing (FWM) and stimulated Raman scattering) and intrachannel [self-phase modulation, intrachannel XPM and intrachannel FWM (IFWM)] nonlinearities, first- and second-order group-velocity dispersion, crosstalk, and intersymbol interference were taken into account (see [10]). The dispersion map, shown in Fig. 1, is composed of 25 spans of length $L=48 \mathrm{~km}$, each span consisting of $2 L / 3 \mathrm{~km}$ of $D_{+}$fiber followed by $L / 3 \mathrm{~km}$ of $D_{-}$fiber. The $Q$-factor is additionally decreased by noise loading. The fiber parameters may be found in [14]. The precompensation of $-320 \mathrm{ps} / \mathrm{nm}$ and corresponding postcompensation are also applied. The simulations were carried out with an average channel power of $0 \mathrm{dBm}$ with a central wavelength of $1552.524 \mathrm{~nm}$ and channel spacing $100 \mathrm{GHz}$. The influence of six neighboring channels on observed channel is considered, because the interchannel

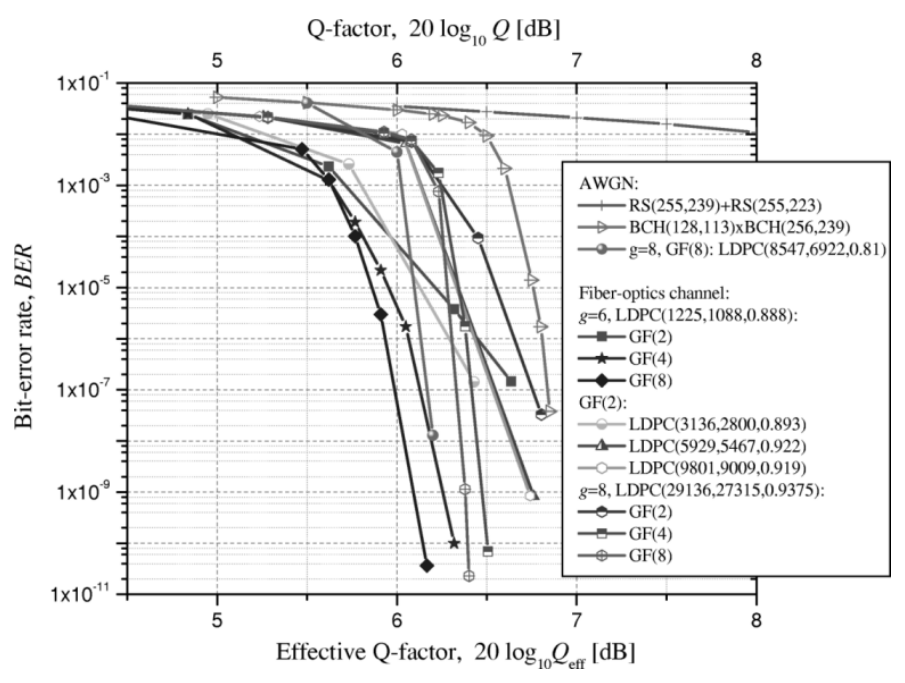

Fig. 2. BER performance of girth-6 $(g=6)$ and girth-8 nonbinary LDPC codes.

nonlinearities from neighboring channels (for dispersion map from Fig. 1) are dominant. The influence of optical filter of bandwidth $2.0 R_{l}$ ( $R_{l}$-the line rate), and electrical filter of bandwidth $0.65 R_{l}$ is also considered. Simulations on other channel models (such as AWGN or chi-square) give too pessimistic predictions of BER [14]. The results for single-channel transmission ignore the influence of interchannel nonlinearities, and as such are not the subject of interest in this letter.

The simulation results on the AWGN channel are given in Fig. 2 (top $x$ axis refers to AWGN, bottom to fiber optics channel model). The girth- 8 lattice $\operatorname{LDPC}(8547,6922,0.81)$ code over $\mathrm{GF}(8)$ is compared against $\mathrm{BCH}(128,113) \times \mathrm{BCH}(256,239)$ turbo product code and $\operatorname{RS}(255,239)+\mathrm{RS}(255,223)$ concatenation scheme. The shorter LDPC code outperforms RS concatenation scheme by $3.14 \mathrm{~dB}$, and turbo product code by $0.7 \mathrm{~dB}$ at BER of $7.75 \cdot 10^{-8}$. Higher coding gains are expected for lower BERs.

The results of simulations for fiber-optics channel model are given in Figs. 2 and 3. Decoding halts when a valid codeword is generated or 25 iterations has been reached. The nonbinary LDPC $(1225,1088,0.888)$ code with $12.59 \%$ redundancy, over $\mathrm{GF}(8)$ provides a coding gain of $9.9 \mathrm{~dB}$ at BER of $10^{-10}$. The improvement over comparable binary $\operatorname{LDPC}(3136,2800$, 0.893) code from the same family [5] is $0.65 \mathrm{~dB}$ at BER of $10^{-7}$. For lower BERs, larger improvement is expected. Further performance improvement is possible by implementing the nonbinary LDPC code over higher dimensional fields. Long LDPC code of rate 0.9375 (overhead only 6.7\%), shown in Fig. 2, provides the coding gain of $9.7 \mathrm{~dB}$ at BER of $10^{-10}$. BER performance of nonbinary block-circulant LDPC codes are shown in Fig. 3. Column-weight-3 code significantly outperforms the column-weight- 2 code. There is no performance loss if the nonzero entries are selected according to (1) instead of being selected randomly. Moreover, from a practical point of view, the block-circulant code facilitates the implementation since the nonzero entries need not be memorized. Block-circulant code of rate 0.864 (with $15.7 \%$ redundancy) over $\mathrm{GF}(8)$ provides a coding gain of $8.3 \mathrm{~dB}$ at $10^{-6}$, outperforming the turbo product code proposal in [4] (with $24.6 \%$ redundancy) by $0.9 \mathrm{~dB}$ (see [4, 


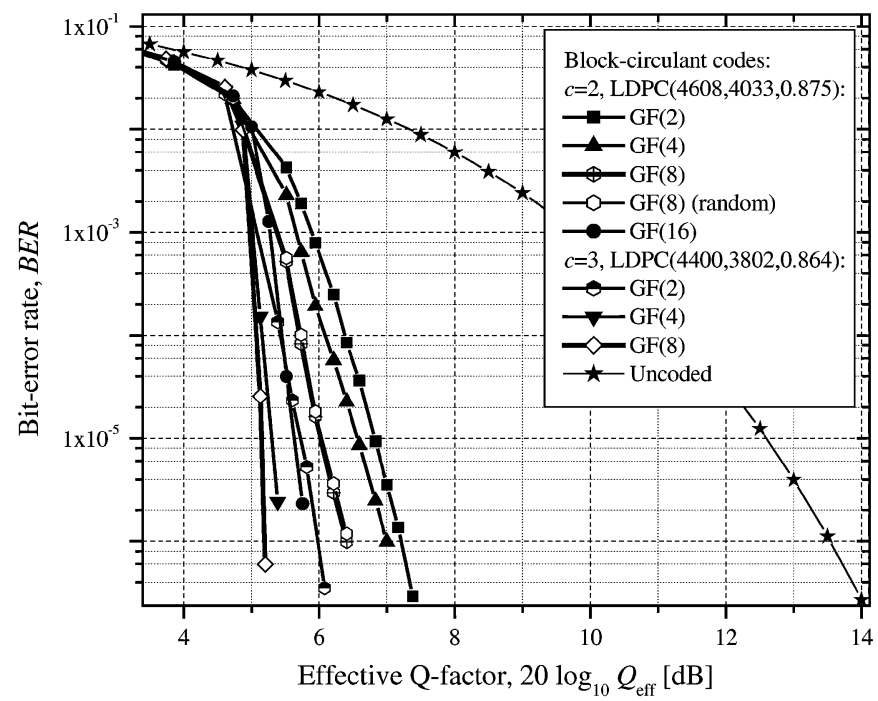

Fig. 3. BER performance of nonbinary block-circulant LDPC codes of column-weight $2(c=2)$ and column-weight $3(c=3)$.

Fig. 6]). Notice that comparison is not completely fair since the influence of IFWM is not included in [4, Fig. 6]. By comparing the curve $\sigma=0.33$ from [4, Fig. 13] and GF(8) curve from Fig. 3, we conclude that the nonbinary LDPC code with $15.7 \%$ redundancy actually outperforms the turbo code with $24.6 \%$ redundancy by $\sim 1.5 \mathrm{~dB}$ at BER of $10^{-6}$. Moreover, the frame size of the turbo product code is significantly larger than that of the nonbinary LDPC code. Much higher coding gains are expected at lower BERs. Therefore, the nonbinary LDPC codes demonstrate high correction capability for error bursts due to Kerr nonlinearities, especially IFWM. The effective $Q$-factor is computed using the expression $Q_{\text {eff }}=\sqrt{2} \operatorname{erfc}^{-1}\left(2 \cdot P_{e, \text { unc }}\right)$, where $P_{e, \text { unc }}$ is the BER of uncoded signal.

The results of simulations are obtained by maintaining a double precision for soft information. In our recent submission [11], we have shown that proper choice in number of quantization bits results in negligible BER performance loss.

The channel capacity of a highly nonlinear channel, determined using finite state machine approach [16], is shown to be strongly dependent on system parameters and channel memory. The plots of the output BER versus input BER for different code rates, commonly reported in literature, are based on Fano's inequality. However, Fano's inequality is valid for discrete memoryless channels only. Since the channel considered in this letter has a memory these curves might be deceiving, and as such are not discussed here.

Based on results by Richardson [12], and our recent study [13], we believe that the proposed codes would not have error floors.

\section{CONCLUSION}

We introduced a class of nonbinary LDPC codes that can achieve a coding gain $9.9 \mathrm{~dB}$ at BER of $10^{-10}$ with overhead of $12.59 \%$ and short length. Similar coding gains can be obtained by employing long irregular binary LDPC codes with optimum node degree distribution. However, highly irregular
LDPC codes are difficult to implement at high bit rates due to high complexity of the decoder. Also, long binary irregular LDPC codes exhibit high processing delay unacceptable for high-speed applications. The structured LDPC codes similar to those proposed in [5], [6], [9]-[11] are more likely to be implemented in practice because their parity-check matrices have simple cyclic or quasi-cyclic structure. Such structures yield to encoders based on shift registers and modulo 2 adders.

The structure of parity-check matrix of block-circulant codes (1) facilitates the implementation because only dimension of permutation matrix and exponents are to be stored (see [15]). In pseudorandom construction proposed in [7], the whole paritycheck matrix, including the content of entries, has to be memorized. Basic operations in lower Galois field are not difficult to implement either [1]. The nonbinary block-circulant LDPC code with $15.7 \%$ redundancy outperforms the turbo code with $24.6 \%$ redundancy by $\sim 1.5 \mathrm{~dB}$ at BER of $10^{-6}$, demonstrating high correction capability of nonbinary LDPC codes for error bursts due to IFWM.

\section{REFERENCES}

[1] S. Lin and D. J. Costello, Error Control Coding: Fundamentals and Applications. Englewood Cliffs, NJ: Prentice-Hall, 1983.

[2] Forward Error Correction for Submarine Systems, Tech. Recomm. G.975/G709. ITU Telecommunication Standardization Sector.

[3] R. M. Pyndiah, "Near-optimum decoding of product codes: Block turbo codes," IEEE Trans. Commun., vol. 46, no. 8, pp. 1003-1010, Aug. 1998.

[4] T. Mizuochi et al., "Forward error correction based on block turbo code with 3-bit soft decision for $10 \mathrm{~Gb} / \mathrm{s}$ optical communication systems," IEEE J. Sel. Topics Quantum Electron., vol. 10, no. 2, pp. 376-386, Mar./Apr. 2004

[5] I. B. Djordjevic and B. Vasic, "MacNeish-Mann theorem based iteratively decodable codes for optical communication systems," IEEE Commun. Lett., vol. 8, no. 8, pp. 538-540, Aug. 2004.

[6] B. Vasic, I. B. Djordjevic, and R. Kostuk, "Low-density parity check codes and iterative decoding for long haul optical communication systems," J. Lightw. Technol., vol. 21, no. 2, pp. 438-446, Feb. 2003.

[7] M. C. Davey and D. J. C. MacKay, "Low-density parity check codes over GF(q)," IEEE Commun. Lett., vol. 2, no. 6, pp. 165-167, Jun. 1998.

[8] H. Wymeersch et al., "Log-domain decoding of LDPC codes over GF (q)," in Proc. IEEE Int. Conf. Commun., vol. 2, Jun. 20-24, 2004, pp. 772-776.

[9] O. Milenkovic, I. B. Djordjevic, and B. Vasic, "Block-circulant lowdensity parity-check codes for optical communication systems," IEEE J. Sel. Topics Quantum Electron., vol. 10, no. 2, pp. 294-299, Mar./Apr. 2004.

[10] I. B. Djordjevic et al., "Projective plane iteratively decodable block codes for WDM high-speed long-haul transmission systems," J. Lightw. Technol., vol. 22, no. 3, pp. 695-702, Mar. 2004.

[11] S. Sankaranarayanan, I. B. Djordjevic, and B. Vasic, "Iteratively decodable codes on $m$-flats for WDM high-speed long-haul transmission," $J$. Lightw. Technol., to be published.

[12] T. Richardson, "Error floors of LDPC codes," in Proc. 41st Allerton Conf. Communications, Control and Computing, Oct. 2003, pp. 1426-1435.

[13] S. K. Chilappagari, S. Sankaranarayanan, and B. Vasic, "Error floors of LDPC codes on binary symmetric channel," IEEE Commun. Lett., submitted for publication.

[14] I. B. Djordjevic, O. Milenkovic, and B. Vasic, "Generalized low-density parity-check codes for optical communication systems," J. Lightw. Technol., vol. 23, no. 5, pp. 1939-1946, May 2005.

[15] M. Mansour, "Implementation of LDPC Decoders," presented at the IEEE Commun. Theory Workshop, Park City, UT, Jun. 13-15, 2005.

[16] I. B. Djordjevic, B. Vasic, M. Ivkovic, and I. Gabitov, "Achievable information rates for high-speed long-haul optical transmission," J. Lightw. Technol., to be published. 